\title{
Design of an Assistant System for Industrial Maintenance Tasks and Implementation of a Prototype Using Augmented Reality
}

\author{
R. Schlagowski ${ }^{1}$, L. Merkel $^{2}$, C. Meitinger ${ }^{1}$ \\ ${ }^{1}$ Faculty of Electrical Engineering, University of Applied Sciences, Augsburg, Germany \\ ${ }^{2}$ Fraunhofer Research Institution for Casting, Composite and Processing Technology IGCV, Augsburg, Germany \\ (claudia.meitinger@hs-augsburg.de)
}

\begin{abstract}
As the complexity of work tasks rises for maintenance workers in modern production facilities, new technologies will be required to support and integrate the service worker of tomorrow. This paper gives an insight into an ongoing research project examining the potential of smart glasses used as a component of assistant systems for workers performing maintenance tasks in an industry 4.0 context. A human centered design process is used to identify the needs of workers and to specify requirements for the assistant system being developed. Thereby, the maintenance of a CNC lathe is used as an example and assistant functions were developed for one specific maintenance task. The architecture of the assistant system proposed in this paper is based on an analysis of the work system including the tasks of the maintenance worker. Finally, the implementation of a first prototype, using state-of-the-art augmented reality smart glasses, is described.
\end{abstract}

Keywords - Assistant System, Maintenance, Augmented Reality, Smart Glasses

\section{INTRODUCTION}

A major goal regarding industrial maintenance within the proclaimed fourth industrial revolution towards more intelligent and connected factories is the transition from classical to predictive maintenance [1, p. 197]. It is characterized by real-time analysis of huge amounts of data which is provided by machinery to determine if it needs to be maintained. Thus it could be possible to maintain every machine on an individual schedule if enough qualified workers were available at any time. Since it is expensive for employers to meet the resulting requirements with traditional human resources, it might be an option to increase the flexibility of workers by improving their ability of learning fast and on demand as well as helping them to provide a larger variety of services with increased efficiency and constant reliability.

This paper suggests assistant systems as appropriate tools to provide information as needed, decrease training time and assist workers during the execution of maintenance tasks. Such assistant systems should not only provide instructions, but also be aware of possible mistakes and hazards. Further, they should not constrain the user physically, for example by occupying the worker's hands, or mentally by increasing their mental workload, e.g. by providing too much information.

\section{METHODOLOGY}

The assistant system is developed on the basis of the human centered design process as described in the standard ISO 9241-210 [2]. Although the developed system should be able to assist the worker while maintaining a great variety of machines in an industrial environment, one specific use case is chosen to validate the potential of the assistant system. The chosen use case is the maintenance of a CNC lathe, which is conducted by an ordinary service worker who has some basic knowledge about industrial maintenance in general, but not about the specific machine.

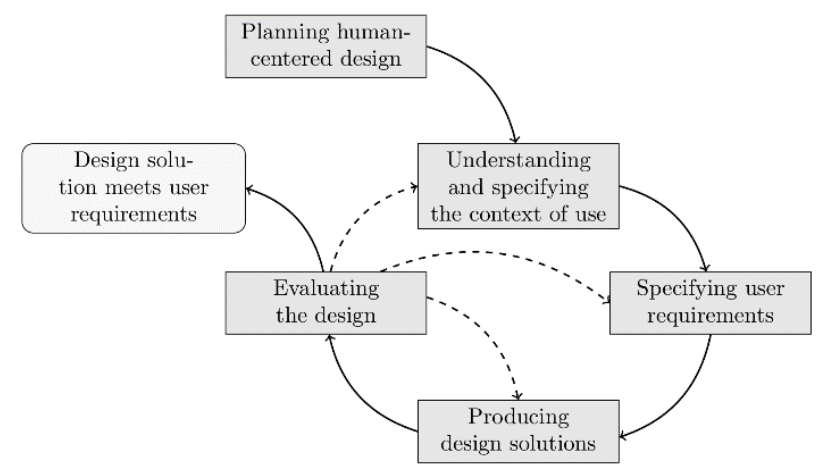

Fig. 1. Human centered design process according to [2]

The first step of the human-centered design process (cf. figure 1) is to examine the context of use. In our project, this is accomplished by analyzing the work system in two versions: the industrial target environment and the test environment in our laboratory. This implies a detailed analysis of the work objective and resulting work tasks of the maintenance worker. Thus, the user requirements can be specified more precisely and new design solutions can be developed in consideration of both the requirements of the specific use case and the resources of the human operator. The prototype that has been realized as a design solution uses state-of-the-art augmented reality technology. It enables a worker to perform a typical maintenance task without any previous training or knowledge in context with the specific model of the lathe. This is achieved by providing step-by-step instructions to the user while simultaneously showing the exact location of the action targets. The prototype will be evaluated in the near future by conducting user studies. Results will be fed back to forthcoming iterations of the human centered design process. 
Paper accepted for 2017 IEEE International Conference on Industrial Engineering and Engineering Management (IEEM). Personal use of this material is permitted. Permission from IEEE must be obtained for all other uses, in any current or future media, including reprinting/republishing this material for advertising or

promotional purposes, creating new collective works, for resale or redistribution to servers or lists, or reuse of any copyrighted component of this work in other works. DOI: 10.1109/IEEM.2017.8289899

\section{RESULTS}

\section{A. Context of Use: Work System Analysis}

According to the standard ISO 6385 [3], a work system consists of one or several human operator(s) using work equipment to fulfill a work objective within a work environment (cf. figure 2). Input and output subsume information, energy and material that are needed or transformed during the work process.

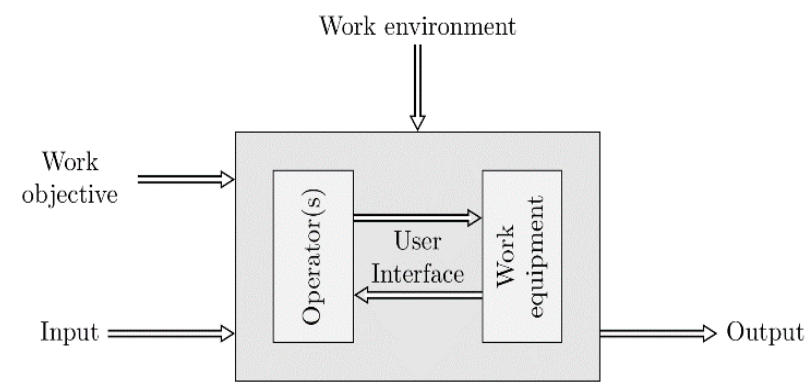

Fig. 2. Illustration of a work system cf. [3]

Table I compares the components of the two variants of the work system under consideration: The industrial scenario which is being aimed for and the test scenario in which development and evaluation take place.

TABLE I

ELEMENTS OF THE CONSIDERED WORK SYSTEMS

\begin{tabular}{rll}
$\begin{array}{r}\text { Work } \\
\text { System } \\
\text { Element }\end{array}$ & Industrial Scenario & Test Scenario \\
\cline { 2 - 3 } $\begin{aligned} \text { Work } \\
\text { Objective }\end{aligned}$ & $\begin{array}{l}\text { Predictive maintenance } \\
\text { of different industrial } \\
\text { machines }\end{array}$ & $\begin{array}{l}\text { Maintenance of a CNC } \\
\text { lathe }\end{array}$ \\
Operator & Service worker & Developer/test subject \\
Work & $\begin{array}{l}\text { Lubrication press, screw } \\
\text { driver, user manual... }\end{array}$ & $\begin{array}{l}\text { Lubrication press, screw } \\
\text { driver, user manual... }\end{array}$ \\
Work & Factory & Fraunhofer LVP \\
Environment & Unput & Unmaintained machines \\
Output & Maintained machines & Maintained lathe \\
\hline
\end{tabular}

${ }^{\mathrm{a}}$ Lernfabrik für vernetzte Produktion (German), English: learning factory for cyber-physical production (operated by Fraunhofer IGCV in Augsburg, Germany)

While input and output, as well as work equipment are quite similar for both scenarios, there are significant differences regarding the operator and the work environment, which need to be considered when specifying the user requirements.

Whereas a real service worker will have some experience with typical tools and maintenance work in general, a test subject might be less experienced in this matter. Furthermore, it is important to consider the test subjects' increased variety in age, education and experience with modern technology that might be a part of the assistant system.
The industrial work environment might not be as clean, quiet, even tempered or well-lit as the test environment. This could lead to difficulties with communication between the user and the assistant system, as well as perception of the environment. There might also be more hazards such as moving objects and the operator might not have as much free space surrounding the machines, which will eventually result in considerable constraints for the freedom of action of the user.

\section{B. Context of Use: Task Analysis}

For the specification of requirements regarding the work objective, it is necessary to analyze and structure maintenance procedures. Industrial maintenance work is often organized as a variety of individual maintenance tasks which need to be executed after a certain amount of working hours, which can be derived from the estimated decrease of the wear stock of affected machine parts [4]. Since these tasks and intervals are specific for every machine model, a classification is required. The approach of this study is to break the maintenance tasks down in two steps, resulting in a classification of subtasks (step 1), and actions (step 2). Every maintenance task consists of subtasks and every subtask of actions. Both might repeat within a variety of different maintenance tasks. Therefore, the amount of building blocks necessary to classify maintenance work is decreased furthermore with every level of classification. Actions are elemental steps, which most humans know as they are part of their everyday life. Subtasks, by contrast, are more specific and contextoriented. As the set of actions they contain is not always known to the human operator, he could be assisted e.g. by providing step-by-step instructions.

Table II shows an excerpt of the results of breaking down the maintenance tasks of the example use case (maintenance of a CNC lathe) into subtasks. Every Task ID represents one maintenance task for the specific machine model as specified by the manufacturer as described in the manual of the machine.

TABLE II

ClASSIFICATION OF SUBTASKS

\begin{tabular}{|c|c|c|c|c|c|c|c|c|c|c|c|}
\hline & \multirow{3}{*}{\begin{tabular}{|l|} 
Time Interval \\
Task ID \\
\end{tabular}} & \multicolumn{10}{|c|}{ Maintenance Tasks } \\
\hline & & \multicolumn{5}{|c|}{$8 \mathrm{~h}$} & \multicolumn{4}{|c|}{$40 \mathrm{~h}$} & \multirow{2}{*}{$\begin{array}{ll}\ldots \\
\ldots\end{array}$} \\
\hline & & 1 & 2 & 3 & 4 & 5 & 6 & 7 & 8 & 9 & \\
\hline \multirow{7}{*}{$\begin{array}{l}\frac{\tilde{v}}{\tilde{J}} \\
\tilde{J} \\
\overrightarrow{0}\end{array}$} & Notification of Warning & $x$ & $x$ & $x$ & $\mathrm{x}$ & $x$ & $\mathrm{x}$ & $x$ & $x$ & $\mathrm{x}$ & $\ldots$ \\
\hline & Opening of Mach. Door & $x$ & $x$ & $x$ & $x$ & $x$ & & $x$ & $x$ & $x$ & $\ldots$ \\
\hline & Optical Inspection & & $\mathrm{x}$ & & & $\mathrm{x}$ & $x$ & & $x$ & $\mathrm{X}$ & $\ldots$ \\
\hline & Removal of Object(s) & & & $x$ & & & $(\mathrm{X})$ & & & & $\ldots$ \\
\hline & Placement of Object(s) & & & $x$ & & & $(X)$ & & & & $\ldots$ \\
\hline & Loosening of Screw(s) & & & & & & $(\mathrm{X})$ & $x$ & & & $\ldots$ \\
\hline & ... & $\ldots$ & $\ldots$ & $\ldots$ & $\ldots$ & $\ldots$ & $\ldots$ & $\ldots$ & $\ldots$ & $\ldots$ & $\ldots$ \\
\hline
\end{tabular}

$(\mathrm{X})$ : Subtask is part of maintenance task under certain conditions

As an example, the maintenance task Optical Control of the Machine Window with the corresponding Task ID \#2, which needs to be executed every eight machine working hours, requires the execution of the subtasks Notification of Warning, Opening of Machine Door and Optical Inspection (amongst others). 
Paper accepted for 2017 IEEE International Conference on Industrial Engineering and Engineering Management (IEEM). Personal use of this material is permitted. Permission from IEEE must be obtained for all other uses, in any current or future media, including reprinting/republishing this material for advertising or

promotional purposes, creating new collective works, for resale or redistribution to servers or lists, or reuse of any copyrighted component of this work in other works. DOI: $10.1109 /$ IEEM.2017.8289899

These subtasks can be broken down even further into actions. These can be identified by observing the execution of subtasks, as conducted by professional service workers, while looking for elemental human actions which can be observed repeatedly. Table III shows an excerpt of the outcome of applying this procedure to our example use case.

TABLE III

CLASSIFICATION OF ATIONS

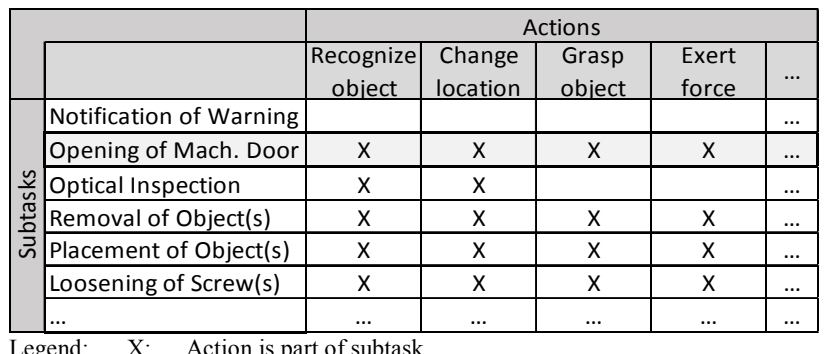

For instance, if the machine door needs to be opened manually, the actions Recognition of an Object (i.e. the machine door), Change of the current Location (i.e. to a position in front of the machine door), Grasp an Object (i.e. the door handle) and Exercising a Force (i.e. pull on the door handle) have to be executed (amongst others).

\section{Specification of User Requirements}

The user requirements can be specified by examining the results of the work system analysis. Table IV lists some user requirements for the industrial scenario of our use case, matched to their corresponding work system element. For the test scenario, the differences as mentioned in section A should be considered.

TABLE IV

USER REQUIREMENTS

\begin{tabular}{ll}
\hline $\begin{array}{l}\text { Element of Work } \\
\text { System }\end{array}$ & User Requirements \\
\hline Work Objective & $\begin{array}{l}\text { Hands-free, system mobility, Predictive } \\
\text { notifications, task execution efficiency, Amount } \\
\text { of human errors, machine variety }\end{array}$ \\
Operator & $\begin{array}{l}\text { Ergonomic standards, multilingualism, } \\
\text { consideration of user qualification/experience, } \\
\text { training efficiency }\end{array}$ \\
Work Equipment & $\begin{array}{l}\text { Information and documentation (of type, } \\
\text { location, application, condition, availability), } \\
\text { variety of supported tools }\end{array}$ \\
Work & $\begin{array}{l}\text { Environmental resistance, network connectivity } \\
\text { and security, safety, required space, illumination }\end{array}$ \\
\hline
\end{tabular}

When formulating the requirements precisely for a specific assistant system, measurable target values must be added to these requirement variables, such as "Task execution efficiency must increase by $10 \%$ " or "The ergonomic standard ISO 9241-110 must be met".

\section{Design Principles}

Assistant systems can be implemented by providing assistant functions which can be assigned to six stages of human action. These stages are "(1) Motivation, activation and goal setting, (2) Perception, (3) Information integration, generating situation awareness, (4) Decision making, action selection, (5) Action execution, and (6) Processing feedback of action results" [5, S. 9]. The user can be assisted in each of these stages separately, by assigning the right assistant functions. For instance, the action "Change location" (cf. Table III) can be assisted by first drawing attention from the operator (Stage 1) and communicating that his or her location must be changed (Stage 2, 3). The next step could be to showcase a variety of possible paths and give advice on which path might be the fastest (Stage 4). Subsequently, the user can be navigated to that location (Step 5). The final assistant function could then be to acknowledge that he or she has arrived (Step 6).

We aim for a cognitive assistant system in particular. A system of this kind focuses on the support of the human operator in his or her effort to successfully achieve the given work objective. This can be achieved by

- directing the attention of the human operator to the currently most important task,

- $\quad$ ensuring that the workload of the human operator is neither too low nor too high, and

- possibly executing subtasks instead of the human operator. [6]

To ensure that the mental workload of the operator is considered correctly, it is important to provide information in an adequate way, so that the operator can perceive it while reliably executing actions at the same time. According to Wickens' model of multiple resources, the performance of actions that are executed in a time-sharing manner will be better if they do not use the same human resources [7]. Thus, for presenting information to the human operator, codes, modalities and placement of information should be chosen in a way, that they won't interfere with the human resources which are currently stressed. Therefore, the assistant system should be able to choose flexibly what kind of resources should be used to not violate this condition. Thus, multiple variations of assistant functions should be implemented for each action that should be assisted.

TABLE $\mathrm{V}$

AVAILABLE HUMAN RESOURCES FOR ASSISTED ACTIONS

\begin{tabular}{|c|c|c|c|c|c|c|c|c|}
\hline & \multirow[b]{2}{*}{ Modality } & \multirow[b]{2}{*}{ Code } & \multirow[b]{2}{*}{ Placement } & \multicolumn{5}{|c|}{ Assisted actions } \\
\hline & & & & $\begin{array}{c}\text { Recognize } \\
\text { position }\end{array}$ & $\begin{array}{l}\text { Change } \\
\text { location }\end{array}$ & $\begin{array}{l}\text { Grasp } \\
\text { object }\end{array}$ & Exert force & \\
\hline \multirow{8}{*}{ 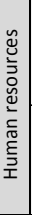 } & \multirow{4}{*}{ visual } & \multirow{2}{*}{$\begin{array}{l}\text { Vocal } \\
\text { Verbal }\end{array}$} & focal & $x$ & $\mathrm{x}$ & $\mathrm{x}$ & $x$ & $\ldots$ \\
\hline & & & ambient & $x$ & $x$ & $x$ & $x$ & $\ldots$ \\
\hline & & \multirow{2}{*}{$\begin{array}{l}\text { Manual } \\
\text { Spatial }\end{array}$} & focal & $\mathrm{x}$ & $\mathrm{x}$ & $\mathrm{x}$ & $\mathrm{x}$ & $\ldots$ \\
\hline & & & ambient & $x$ & $x$ & $x$ & $x$ & $\ldots$ \\
\hline & \multirow{4}{*}{ auditory } & \multirow{2}{*}{$\begin{array}{l}\text { Vocal } \\
\text { Verbal }\end{array}$} & focal & $x$ & $x$ & $x$ & $x$ & $\ldots$ \\
\hline & & & ambient & & & & & $\ldots$ \\
\hline & & \multirow{2}{*}{$\begin{array}{c}\text { Manual } \\
\text { Spatial }\end{array}$} & focal & & & & & $\ldots$ \\
\hline & & & ambient & $\mathrm{x}$ & $\mathrm{x}$ & & & \\
\hline
\end{tabular}

Legend: $\quad \mathrm{X}$ : Hardware can use human resource to assist execution of action 
Table $\mathrm{V}$ shows human resources, which the available hardware (smart glasses) can make use of while assisting the operator performing actions as classified in chapter B. In the final assistant system, as many of these variations as possible should be implemented.

\section{E. Design Solution}

An assistant system architecture as shown in figure 3 is used to provide assistant functions. It consists of modules that are based on typical steps of human information processing as e.g. described by the Cognitive Process $[6,8]$ :

- Sensation of the environment: Acquisition of data about the situation the operator currently is in. This includes the operator's input, machine data and the registration of the active maintenance task.

- Interpretation of the situation: Interpretation of the situational context to achieve situation awareness, e.g. consideration of the subtask the operator should execute next, his or her condition and whether there are any hazards that need to be taken into account.

- Determination of goals: The assistant system has to decide, which goals it should pursue. This is especially important in case of goals competing against each other (e.g. support the execution of the next sub-task / stop work process due to safety issues).

- Action planning: Planning on how the operator will be assisted, e.g. by presenting needed information including the choice of code, modality, placement of information and assisted stage(s) of human action or by executing actions on behalf of the human operator.

- Execution of actions: Provision of assistant functions to the operator as previously planned.

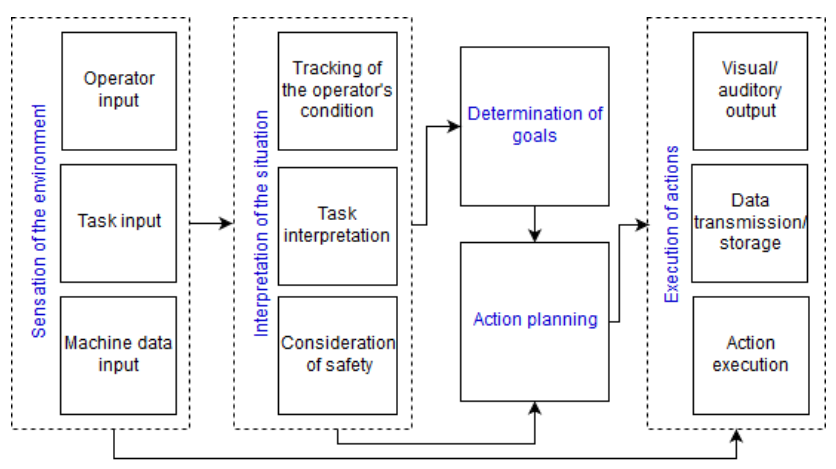

Fig. 3. Architecture of the assistant system

When applied to our example use case, the maintenance task Optical Control of the Machine Window is registered and machine data like passed working hours or machine door status is tracked in real time together with the operator's input such as gaze direction or user location. This information is then processed to understand the work environment and the situational context, e.g. the current subtask and action that is being executed at the moment, like Opening of Machine Door and Change of Location in an industrial environment, maybe close to a transportation route. Although the subsequent action Grasp Object could be assisted subsequently e.g. by showing the location of the target object, the assistant system might recognize a possible threat like an approaching transport vehicle and may therefore decide to warn the user first within the architecture component Determination of Goals. The next step for the system is to plan how the operator should be warned, respectively which stages of human action should be assisted by using the right codes, modalities and placement. In this example, the system may choose to draw user attention by playing back a relatively loud acoustic warn ing signal through both stereo speakers (Stage of human action: (1), Modality: auditory, Code: manual spatial, Placement: focal), followed up by the playback of a voice telling the user to stop moving (Stage of human action: (4), Modality: auditory, Code: vocal verbal, Placement: focal).

\section{F. Prototype}

To implement the described assistant system architecture, a prototype was developed. As hands-free and multi-modal interaction between the operator and the assistance system are important user requirements for industrial maintenance work, the state-of-the-art augmented reality smart glasses HoloLens by Microsoft (cf. figure 4) were chosen as a hardware component. The used SDK for this prototype includes the game engine Unity and software libraries/Unity Prefabs by Microsoft and Vuforia.

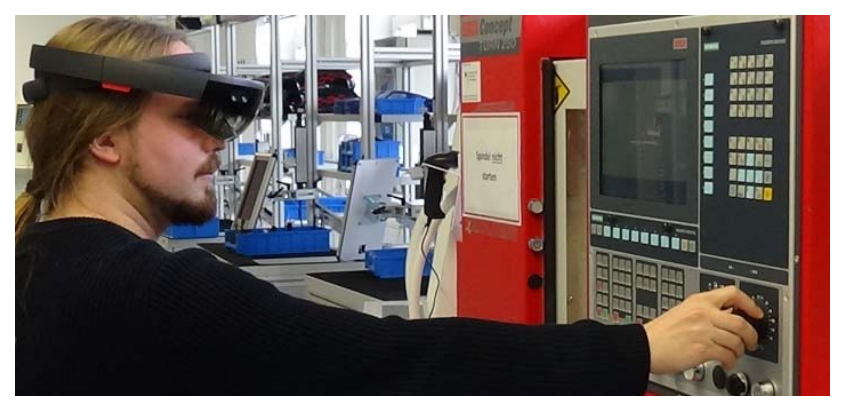

Fig. 4. Test of the first prototype using Microsoft HoloLens

After having chosen a maintenance task from the main menu, the prototype is able to assist the user by displaying instructions for subtasks and actions in the exact location of the action target by using an augmented reality graphic user interface. As soon as an action is done, the user needs to use voice or gesture commands to proceed to the next step. The progress of the task is shown in a static graphic user interface. Furthermore, pointing arrows are implemented to show the direction of machine elements that are not within the limited field of view.

The components of the architecture described in section E, are implemented in Game Objects in Unity, which derive methods from parent classes as defined in attached C\# Scripts. Table VI shows which functional Game Object in the software design of the prototype corresponds to the architecture components as shown in figure 3. For example, displaying instructions by the use of an augmented reality user interface is implemented by the 
Paper accepted for 2017 IEEE International Conference on Industrial Engineering and Engineering Management (IEEM). Personal use of this material is permitted. Permission from IEEE must be obtained for all other uses, in any current or future media, including reprinting/republishing this material for advertising or

promotional purposes, creating new collective works, for resale or redistribution to servers or lists, or reuse of any copyrighted component of this work in other works. DOI: $10.1109 /$ IEEM.2017.8289899

WorldCanvas object. The StaticCanvas object is used to display information in the operator's field of view and the MachineElements objects are used to display holograms on top of targeted machine parts by using augmented reality. All three components are part of execution of actions (cf. Table VI) and have most of their logic implemented in attached (...Behaviour) C\# scripts.

TABLE VI

COMPONENTS OF PROTOTYPE IMPLEMENTATION

\begin{tabular}{|c|c|c|}
\hline \multirow{2}{*}{$\begin{array}{l}\text { Architecture } \\
\text { component } \\
\text { (cf. figure 3) }\end{array}$} & \multicolumn{2}{|c|}{ Implementation } \\
\hline & $\begin{array}{l}\text { Unity Game } \\
\text { Objects }\end{array}$ & C\# Scripts (*.cs) \\
\hline $\begin{array}{l}\text { Sensation of the } \\
\text { Environment: Operator } \\
\text { input }\end{array}$ & $\begin{array}{l}\text { HoloLensInputMo } \\
\text { dule }^{1} \text {, } \\
\text { SpatialMapping }^{1} \text {, } \\
\text { Cursor }\end{array}$ & $\begin{array}{l}\text { SpeechManager }{ }^{1} \text {, } \\
\text { GazeGestureManager }^{1}\end{array}$ \\
\hline $\begin{array}{l}\text { Sensation of the } \\
\text { Environment: Machine } \\
\text { data input }\end{array}$ & $\begin{array}{l}\text { ARCamera }^{2} \text {, } \\
\text { VuMarks, Anchor }\end{array}$ & $\begin{array}{l}\text { VuforiaBehaviour }{ }^{2} \text {, } \\
\text { VuMarkBehaviour }^{2} \text {, } \\
\text { AnchorBehaviour }\end{array}$ \\
\hline $\begin{array}{l}\text { Sensation of the } \\
\text { Environment: Task input }\end{array}$ & $\begin{array}{l}\text { TaskManager, } \\
\text { MainCanvas }\end{array}$ & $\begin{array}{l}\text { StartUpStepHandler, } \\
\text { TaskManagerBehaviour }\end{array}$ \\
\hline $\begin{array}{l}\text { Interpretation of the } \\
\text { Situation, Determination } \\
\text { of goals, Action } \\
\text { planning }\end{array}$ & $\begin{array}{l}\text { MaintenanceTask } \\
\text { 01, DemoTask }\end{array}$ & TaskStepHandler \\
\hline Execution of actions & $\begin{array}{l}\text { MachineElements, } \\
\text { WorldCanvas, } \\
\text { StaticCanvas }\end{array}$ & $\begin{array}{l}\text { MachineElementBehaviour, } \\
\text { WorldCanvasBehaviour, } \\
\text { StaticCanvasBehaviour }\end{array}$ \\
\hline
\end{tabular}

As this first functional prototype doesn't consider the safety situation of the user, the goals and the next action can always be directly derived from the component Interpretation of the Situation.

\section{DISCUSSION}

Early tests of the prototype showed a great potential of Microsoft HoloLens for assistant functions within the fields of perception and information integration/situation awareness (cf. stages of human action, section D.). As these tests were only conducted on a small scale, larger user studies need to be performed to proof this potential and compare the usability of the prototype features. Furthermore, the missing components of the conceptual architecture need to be realized in future implementations and other hardware needs to be integrated and tested. The reliability of the system can also be increased by recognizing the execution and the quality of a performed step automatically, for example by using precise optical tracking. Further goals are to realize the consideration of the abilities and knowledge of the individual user and increase the variety of supported machines. Big data concepts (e.g. cloud computing) can also be implemented to improve the acquisition of data and the prediction of due maintenance work. The system's increased situation awareness will then allow for more complex action planning and goal determination algorithms to be implemented.

\section{CONCLUSION}

This paper presented the design and development of a prototype for assisting the execution of maintenance tasks in an industrial environment, based on an architecture which was developed by using a human centered design process, which includes a work system analysis and a structured decomposition of maintenance tasks. This first prototype enables the user to perform maintenance tasks without prior knowledge, so that it can be stated that the concept of hands-free assistant systems using augmented reality have the potential to aid the maintenance worker of the future significantly. Still, it leaves plenty of room for improvement, as there are user requirements that need to be fulfilled by completing further implementation, iteration and evaluation procedures. This includes automatic detection of the operator's performed actions and his or her condition. Furthermore, enhanced acquisition of machine data is required to consider safety issues and goals. As time will progress, new hard- and software solutions will also be made available to realize better systems, that can offer a larger variety of assistant functions while satisfying the ergonomic needs of the future worker.

\section{ACKNOWLEDGMENT}

We thank the Bavarian Ministry of Economic Affairs and Media, Energy and Technology for funding the project 'Digitalisierte Produktion - Demonstrations- und Trainingszentrum für Cyber-Physische Produktionssysteme' within the program 'Bayern Digital'.

\section{REFERENCES}

[1] U. Kumar, A. Ahmadi, A. K. Verma, and P. Varde, Current Trends in Reliability, Availability, Maintainability and Safety. Cham: Springer International Publishing; 2016.

[2] DIN, Ergonomics of human-system interaction - part 210: Human-centered design for interactive systems (ISO 9241210:2010); Berlin: Beuth Verlag; 2010.

[3] DIN, Ergonomics principles in the design of work systems (ISO 6385:2016); Berlin: Beuth Verlag; 2014.

[4] DIN, Fundamentals of maintenance (DIN 31051:2012-09); Berlin: Beuth Verlag; 2012.

[5] H. Wandke. Assistance in human-machine interaction: A conceptual framework and a proposal for a taxonomy, Theoretical Issues in Ergonomics Science; 2005, vol. 6, no. 2, pp. 129-155.

[6] R. Onken \& A. Schulte. System ergonomic design of cognitive automation: dual mode cognitive design of vehicle guidance and control work systems. Berlin, Heidelberg: Springer; 2010.

[7] C. D. Wickens. Multiple resources and mental workload. Human Factors; 2008, 50(3): 449-455.

[8] H. Putzer \& R. Onken. COSA - a generic cognitive system architecture based on a cognitive model of human behaviour. Cognition Technology and Work; 2003, 5, pp. 140-151. 